Revue

de Sémantique

et Pragmatique
Revue de Sémantique et Pragmatique

39 | 2016

Varia

\title{
Prédiscours, interdiscours et postdiscours : analyse critique de la circulation des possibles discursifs
}

Albin Wagener

\section{(2) OpenEdition \\ Journals \\ Édition électronique \\ URL : http://journals.openedition.org/rsp/422 \\ DOI : $10.4000 /$ rsp. 422 \\ ISSN : 2610-4377 \\ Éditeur \\ Presses universitaires d'Orléans}

Édition imprimée

Date de publication : 1 octobre 2016

Pagination : 95-110

ISSN : 1285-4093

\section{Référence électronique}

Albin Wagener, «Prédiscours, interdiscours et postdiscours : analyse critique de la circulation des possibles discursifs », Revue de Sémantique et Pragmatique [En ligne], 39 | 2016, mis en ligne le 01 octobre 2017, consulté le 20 avril 2019. URL : http://journals.openedition.org/rsp/422 ; DOI : 10.4000/ rsp. 422 


\title{
PRÉdISCOURS, INTERDISCOURS ET POSTDISCOURS : ANALYSE CRITIQUE DE LA CIRCULATION DES POSSIBLES DISCURSIFS
}

\author{
Albin Wagener \\ IMIE / CoDiRe (EA 4643 - Université de Nantes)
}

\section{ANALYSE CRITIQUE DU DISCOURS : UNE DÉFINITION.}

Afin de pouvoir proposer une théorie susceptible de mettre en dynamique prédiscours, interdiscours et postdiscours, il parait avant tout élémentaire de proposer une définition du discours qui puisse permettre de soutenir nos hypothèses. Cette définition du discours pourra ensuite fournir un socle stabilisé pour la construction d'une théorie d'analyse critique du discours basée à la fois sur la sémantique, la pragmatique et une approche cognitive des développements discursifs. Ainsi, notre position concernant une définition du discours s'inscrit dans la tradition pragmatique anglo-saxonne, telle que proposée notamment par Norman Fairclough (2005 : 915-916) :

«J'utilise le terme 'discours' pour qualifier les éléments linguistiques et sémiotiques (comme par exemple les productions visuelles et le 'langage corporel') du monde social, mais je l'utilise également dans une optique relationnelle, en mettant l'accent sur les relations entre les éléments linguistiques et sémiotiques du monde social et d'autres éléments, y compris matériels » ${ }^{1}$

1 "I use the term 'discourse' for linguistic and other semiotic elements (such as visual images and 'body language') of the social, but I use it in a relational way, with a focus on relations between linguistic/semiotic elements of the social and other (including material) elements", notre traduction. 
Tout comme Fairclough, nous estimons que les discours ne sont pas uniquement construits et représentés à travers les éléments linguistiques ou verbaux seuls, mais que ceux-ci sont tout autant alimentés par des items sémiotiques pluriels, utilisant le sens afin de pouvoir soutenir la construction et l'évolution de sens nouveau. Une telle définition du discours permettra, comme nous le verrons, d'élargir non seulement la notion d'interdiscursivité, mais également de pouvoir étendre l'analyse de discours critique à des objets plurisémiotiques, telles que les publications en ligne par exemple (Wagener, 2014), afin de pouvoir appréhender des données de production de sens les plus complètes et complexes possibles. De surcroît, une acception large du discours répond également aux opérations cognitives auxquelles les individus restent soumis afin de pouvoir accéder à la réception, l'interprétation, la production et la reproduction du sens - des tâches résolument complexes et socialement nourries, déjà évoquées par Patrick Charaudeau (1995) et précisées ici par Teun Van Dijk (1993 : 257) :

« La cognition sociale telle que nous la comprenons est définie par des représentations d'arrangements sociétaux, de groupes et de relations socialement partagées, ainsi que des opérations mentales comme l'interprétation, la pensée et l'argumentation, l'inférence et l'apprentissage, entre autres. (...) Le discours, la communication et toute autre forme d'action et d'interaction ne peuvent s'opérer sans cognition sociale. $»^{2}$

Ainsi, une acception plurisémiotique du discours, telle que proposée par Fairclough, permet précisément à l'intuition de Van Dijk à propos de la cognition sociale de pouvoir opérer de manière plus large et plus complète ; une telle définition nous sera d'autant plus utile dans une théorie croisée et complexe des prédiscours, des interdiscours et des postdiscours, dans la mesure où les représentations, génératrices de cognition sociale et générées par la cognition sociale, constituent tout autant la source, le carburant et le résultat des discours. Le positionnement que nous soutenons, orienté vers un discours plurisémiotique (donc linguistique, mais pas exclusivement) et vers une inscription de la cognition sociale comme mécanique de lien entre discours, représentations et contextes sociétaux, nous enjoint à adopter une définition de l'analyse critique du discours qui permettrait justement de pouvoir analyser ce que Seyla Benhabib nomme les réseaux de récits (2002 : 6-7), mais également d'ancrer les processus discursifs dans les réalités sociales, comme le proposent également Norman Fairclough et Ruth Wodak (1997 : 258) :

2 "Socially shared representations of societal arrangements, groups and relations, as well as mental operations such as interpretation, thinking and arguing, inferencing and learning, among others, together define what we understand by social cognition. (...) Discourse, communication and (other) forms of action and interaction are monitored by social cognition", notre traduction. 
«L'analyse critique de discours définit le discours (...), comme une forme de 'pratique sociale'. Décrire le discours comme une pratique sociale implique une relation dialectique entre un événement discursif spécifique et les situations, institutions et structures sociales qui le cadrent : elles forment l'événement discursif, mais il les forme également en retour. Ainsi le discours est socialement constitutif et socialement conditionné ; il élabore les situations, les objets de savoir, ainsi que les identités sociales et les relations des individus et groupes d'individus. (...) Par la manière dont elles représentent les objets et positionnent les individus, les pratiques discursives peuvent avoir d'importants effets idéologiques, en ce qu'elles permettent la production et la reproduction de relations de pouvoir inégales, par exemple entre les classes sociales, les femmes et les hommes, et les majorités et minorités ethniques ou culturelles $»^{3}$

Cependant, notre inscription dans une tradition pragmatique et sociale de l'analyse discursive anglo-saxonne se trouve également reliée à la linguistique de tradition française, en ce que celle-ci permet, selon nous, de développer des cadres théoriques et méthodologiques plus complets et plus structurés. Ainsi donc, une définition de l'analyse critique du discours est bien sûr essentielle, mais si tout peut être discours, il faut également comprendre dans quelle mesure un discours isolé, recueilli et étudié peut réellement devenir un objet scientifique, au sens d'item susceptible d'être analysé et de permettre au chercheur de formuler des hypothèses. C'est exactement ce que propose Julien Longhi (2009 : 90) en orientant les objets discursifs vers la construction du sens de manière phénoménologique :

«Le concept d'objet discursif, en ce qu'il articule les domaines de la sémantique et la linguistique du discours, constitue un moyen d'accès à la construction du sens en discours. Cette construction, dans une approche phénoménologique, est intimement liée à la perception, et se constitue dynamique à travers l'activité discursive. Le sens est alors décrit comme un parcours, au cours duquel l'agrégation des éléments qui le constituent s'élabore à travers certaines phases. En outre, ces phases sont liées à la dynamique du corpus, et s'anticipent les unes les autres. (...) Les visées discursives sont constitutives de l'activité de nomination, et cela se retrouve dans la mise en rapport des objets et avec le sens dont ils sont crédités »

3 "CDA sees discourse (...) as a form of 'social practice'. Describing discourse as social practice implies a dialectical relationship between a particular discursive event and the situation(s), institution(s) and social structure(s), which frame it: the discursive event is shaped by them, but it also shapes them. That is, discourse is socially constitutive as well as socially conditioned - it constitutes situations, objects of knowledge, and the social identities of and relationships between people and groups of people. (...) Discursive practices may have major ideological effects - that is, they can help produce and reproduce unequal power relations between (for instance) social classes, women and men, and ethnic/cultural majorities and minorities through the ways in which they represent things and position people", notre traduction. 
L'apport fondamental de Longhi concernant les objets discursifs nous invite justement à permettre le découpage des flux discursifs (en tant que courants plurisémiotiques et socialement actifs) en objets distincts, qui s'inscrivent à la fois dans une dynamique diachronique (les objets discursifs se succèdent dans le temps et font écho les uns aux autres) et synchronique (les objets discursifs co-émergent en fonction d'environnements à la fois différents mais irrémédiablement reliés), tout en nourrissant entre eux des rapports bien spécifiques, obéissant à des modalités que nous allons dès à présent décrire, à travers une définition des notions d'interdiscours, de prédiscours et de postdiscours, afin d'élaborer une proposition d'analyse critique pluridiscursive.

\section{PRÉSENTATION DES NOTIONS D'INTERDISCOURS, DE PRÉDISCOURS ET DE POSTDISCOURS.}

Présente depuis plusieurs années déjà en linguistique de tradition française, la notion d'interdiscours a d'abord été proposée par Michel Pêcheux (1975) et a depuis fait l'objet de nombreux articles, non seulement en écho aux travaux de Pêcheux, mais également afin d'affiner, d'approfondir et d'appliquer la notion à des objets d'étude divers. Ainsi, la dynamique de l'activité discursive se retrouve notamment mise en exergue dans la définition d'une zone interdiscursive réactualisée, proposée par Nathalie Garric et Julien Longhi, comme autant d'espaces pluriels, multimodaux et socialement référencés (2013:65) :

«Les discours n'appartiennent pas à des zones de pratiques délimitées. Situés dans l'interdiscours, considéré comme espace de circulation dynamique et conflictuel, ils sont traversés et investis par des objets sociaux qui prennent sens dans la pluralité des trajets interprétatifs auxquels participe le sujet en assumant différents rôles sociodiscursifs successifs. Il est tantôt consommateur potentiel, tantôt électeur potentiel, tantôt citoyen actif ou retraité »

Ainsi, l'interdiscours pourrait constituer à la fois le point de passage d'objets sociaux et l'espace de transition d'objets discursifs (voire de fragments d'objets discursifs), tout en étant soumis aux forces parfois chaotiques des trajets interprétatifs. Dans une telle optique, la subjectivité des porteurs, producteurs, interprétants et récepteurs de discours devient capitale : les locuteurs eux-mêmes deviennent les agents incontournables de la circulation du sens dans un environnement social riche et complexe. En d'autres termes, ce sont les sujets eux-mêmes, en tant qu'agents et acteurs sociaux, qui deviennent par leur existence même les conditions essentielles à la fois des trajets interprétatifs des objets discursifs, ainsi que des passages possibles au sein des zones interdynamiques interdiscursives. Pour aller plus loin, Nathalie Garric et Valérie Capdevielle-Mougnibas proposent précisément l'inscription de la subjectivité, voire même de l'intersubjectivité, comme base fondamentale de l'existence de l'interdiscursivité (2009: 108) : 
«La discursivité est indissociable de l'interdiscursivité qui définit le sujet en tant que subjectivité construite par des rapports de codétermination socio-idéologiques (places sociales) et génériques (places énonciatives). Le sujet discursif n'est ni tout individuel, ni tout intentionnel, ni tout surdéterminé. Relevant du registre, il en reçoit les déterminations identitaires, idéologiques et collectives ; relevant d'une formation discursive, il est sujet (social), singulier et critique, construit dans la différenciation »

En arguant ainsi d'un positionnement ontologiquement incontournable du sujet dans la dynamique interdiscursive, Garric et Capdevielle-Mougnibas situent selon nous de facto l'interdiscursivité dans l'intersubjectivité, en tant qu'espace social de circulation et comme lieu de l'intime (Wagener 2015 : 220), puisque le sujet socialement situé partage nécessairement sources, références et possibles discursifs avec ses congénères, mais également parce que, dans une forme d'intrasubjectivité, le sujet social et activement discursif doit pouvoir mobiliser, de façon cognitivement pertinente, des références discursives variablement ancrées dans le temps et dans l'espace synchronique immédiat. En miroir de cela, les groupes sociaux ou les sociétés en général fonctionnent sur une intersubjectivité qui permet l'émergence du collectif et de la circulation des références communes. A travers cela, l'interdiscours devient un espace au sein duquel il est impossible de se mouvoir sans comprendre la prégnance des opérations cognitives et sociales. C'est précisément à partir de ce moment qu'au sein des réseaux des opérations interdiscursives, la notion de prédiscours nous semble à la fois opérationnellement utile et épistémologiquement pertinente afin d'aller plus avant dans la construction d'une analyse critique pluridiscursive. En tant qu'opérateurs cognitivement actifs, les prédiscours, tels que développés par Marie-Anne Paveau, permettent en effet de comprendre les mécanismes de circulation de sens au sein des espaces interdiscursifs (2006 : 14) :

«Les prédiscours sont en effet des opérateurs dans la négociation du partage, de la transmission et de la circulation du sens dans les groupes sociaux. Je les définis comme un ensemble de cadres prédiscursifs collectifs qui ont un rôle instructionnel pour la production et l'interprétation du sens en discours. J'entends par prédiscours des contenus sémantiques (au sens large de culturel, idéologique, encyclopédique), c'est-à-dire des savoirs, des croyances et des pratiques. (...) Ces cadres ne gisent pas seulement dans la tête des individus et dans la culture des groupes, mais sont distribués dans les contextes matériels de la production discursive, ce qui explique pourquoi j'y intègre les pratiques, à côté des savoirs et des croyances qui sont d'ordre représentationnel »

Selon nous, le rôle précisément instructionnel des prédiscours est ici capital si l'on souhaite comprendre la manière dont les prédiscours peuvent être utilisés par les locuteurs. En d'autres termes, pour paraphraser Paveau, les prédiscours constituent des ressources sémantiques multiples, plurielles et interconnectées (donc résolument interdiscursives) mobilisables à tout moment, en fonction des 
situations. Ces prédiscours pourraient d'une certaine manière être comparés à des briques discursives, permettant certes aux locuteurs de construire euxmêmes leurs discours à l'aide de ces briques de tailles et de couleurs différentes, mais toujours dans les limites des formes que les prédiscours mettent à leur disposition. Pour résumer, chaque locuteur pourrait alors bien sûr fabriquer des discours qui lui sont propres, en fonction des situations dans lesquelles il se retrouve, mais à partir d'atomes discursifs préconstruits et formatés dans un environnement social, psychologique, économique, linguistique et politique précis ; nous pourrions alors arguer du fait que le discours constitue une molécule dont les prédiscours constituent les atomes élémentaires, combinés par le génie des locuteurs, en fonction de leurs représentations et de leur perception des contextes. Cependant, une telle acception des prédiscours demeure insuffisante si nous souhaitons comprendre la manière dont les individus eux-mêmes opèrent et sélectionnent les prédiscours qu'ils souhaitent employer, combiner ou tronquer en fonction des situations. Pour aller plus loin, une plongée dans les processus cognitifs reste incontournable ; en effet, pour que les locuteurs puissent avoir accès à ces prédiscours, il faut qu'ils puissent nourrir et se laisser influencer par des représentations qui circulent dans l'espace social au sens large. Ceci ne signifient pas qu'ils aient conscience de cet accès ou qu'ils aient l'intention de les mobiliser, mais que l'opération discursive passe nécessairement par un sujet discursif, au moins comme terre d'émergence de production discursive. Pour Paveau, les représentations sont précisément à l'origine des distributions cognitives à l'œuvre pour les locuteurs (2006:56) :

« Je définis alors la représentation comme une entité cognitive (la représentation est un organisateur mental) qui fournit à l'individu un mode d'être en société (la représentation est une forme de connaissance du monde) construit ou activé en discours (la représentations est formulée discursivement, de manière implicite ou explicite) »

Il devient alors important de pouvoir relier les prédiscours aux interdiscours tels que postulés par Garric et Longhi. Pour cela, nous proposons le schéma suivant, que nous souhaitons par ailleurs commenter :

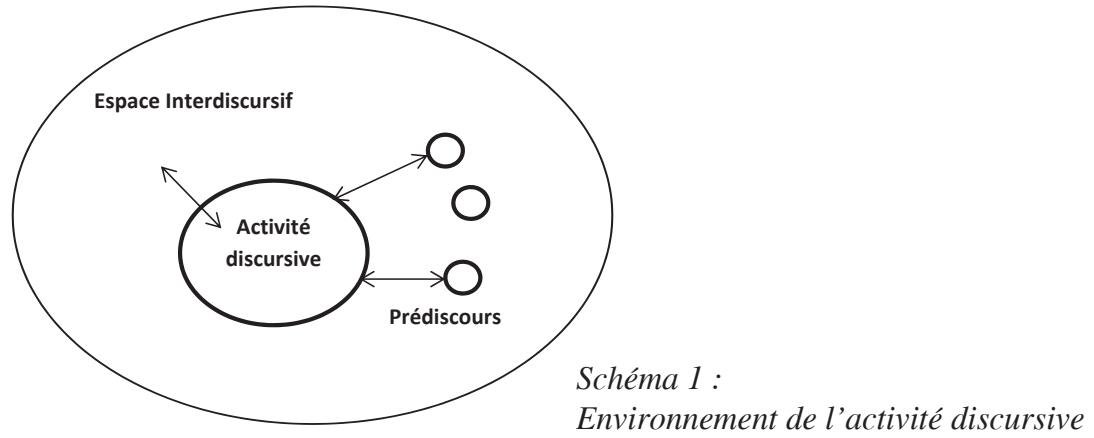


Nous postulons le fait que l'activité discursive émerge naturellement de l'espace interdiscursif, que nous envisageons ici dans son ensemble complexe, regroupant à la fois les environnements individuels (cognitifs, émotionnels, psychologiques), sociaux, éducatifs, sociétaux, politiques, économiques et linguistiques. Au sein de cet espace interdiscursif, des cadres prédiscursifs sont à disposition du locuteur qui va produire une activité discursive ; il peut les mobiliser ou non, sans qu'une intentionnalité soit nécessaire, tout en sachant que ces prédiscours vont nourrir l'activité discursive, tout comme cette activité va également les nourrir en retour. Cependant, ces prédiscours sont mobilisables au sein de l'activité discursive uniquement s'il existe un lien représentationnel, capable de faire sens entre l'activité discursive et les prédiscours disponibles. Il est également important de souligner le fait que l'activité discursive se situe elle-même dans l'espace interdiscursif et ne saurait s'en soustraire : elle l'influence autant qu'elle est influencée par cet espace. Nous avons pour le moment volontairement évacué la notion de postdiscours, que nous pourrions définir de la façon suivante (non pas pour la figer, mais pour pouvoir mieux la qualifier), et que nous réintégrerons ultérieurement dans un autre schéma : les postdiscours constituent les possibles répercussions de l'activité discursive, dotées de potentiel évolutif, au sein de l'espace interdiscursif et sur les prédiscours eux-mêmes, à savoir :

- Les éventuelles modifications des prédiscours suite à l'activité discursive

- Les impacts possibles de l'activité discursive sur la constitution de l'espace interdiscursif, y compris l'apport de nouveaux prédiscours

- Les possibles discursifs, en écho aux possibles argumentatifs (Galatanu, 2009) qui pourront émerger suite à l'accomplissement de l'activité discursive

En d'autres termes, l'introduction des postdiscours devient utile si l'on souhaite pouvoir, dans l'esprit de l'analyse critique du discours, mesurer les impacts possibles et les potentiels actanciels des discours et des sujets, tout en tentant de cerner les évolutions et dynamiques socialement et individuellement possibles. Par exemple, suite à un discours politique ou institutionnel, il peut être intéressant d'imaginer les postdiscours putatifs afin de pouvoir mesurer et isoler les conséquences discursives possibles ; pour ce faire, il faut cependant pouvoir mieux comprendre la façon dont sont liés entre eux prédiscours, espace interdiscursif et activité discursive. Pour ce faire, il nous semble capital de revenir à la façon dont le sens peut circuler entre les opérateurs discursifs. 


\section{HYBRIDATION À PARTIR DES NOTIONS DE SENS COMMUN ET DE NCUDS SÉMANTIQUES.}

Depuis plusieurs années maintenant, les travaux de Georges-Elia Sarfati développent de manière structurée une théorie du sens commun qui s'articule à la fois selon un axe sémantique et un axe pragmatique, en lien avec des communautés de discours qui partagent des sens communs. Ainsi, Sarfati précise également que ces liens ont vocation à être modifiés de manière diachronique et synchronique (2007: 76), dans l'esprit de l'analyse critique qui est la sienne :

« le [sens commun] d'une communauté de discours n'est jamais fixé une fois pour toutes ; il est susceptible de varier aussi bien en synchronie qu'en diachronie, d'une part du fait de son historicité foncière, d'autre part du fait de sa plasticité constitutive : ce qui est principalement en jeu dans les mutations d'une communauté de sens c'est le remaniement ou la duplication de sa topique directrice et régulatrice »

Le principe même des communautés de discours et de la plasticité du sens commun qui y circule fait directement écho à la nécessaire intersubjectivité des espaces interdiscursifs définis par Garric et Longhi. La théorie du sens commun est par ailleurs complexe, dans la mesure où le sens commun lui-même est généré en fonction de processus précis (Sarfati, 2011 : 146), qui motivent une communauté à maintenir le sens en question et de lui permettre d'opérer dans des contextes donnés. Dans ce sens, la théorie du sens commun s'articule sur trois niveaux, que nous nous proposons de résumer ici, en espérant ne pas les simplifier à outrance :

- Le canon, niveau de spécialisation (exemple : le scientifique spécialiste)

- La vulgate, niveau de connaissance (exemple : l'amateur ou le connaisseur)

- Enfin un double niveau, celui de la doxa (où l'on fonctionne par « ouï dire ») et de l'idéologisation (où l'on fonctionne par simplification avec effets ciblés)

Ces trois niveaux ne peuvent être analysés que de manière incomplète si l'on ne fait pas référence à la notion d'institution de sens, que Sarfati détaille de la façon suivante (2011: 144) :

" [l'] ensemble des formes ou des structures sociales édifiées en vue de régir, d'organiser et de sanctionner le développement des pratiques collectives ; par ext. formes ou structures sociales destinées à remplir certaines fonctions collectives. Les institutions diffèrent entre elles par le type de savoir et de discours qu'elles mettent en œuvre à des fins d'efficacité interne et externe. (...) L'idée d'institution de sens vise spécifiquement l'analyse de la formation (c'est-à-dire le mode d'articulation du sens produit par l'institution considérée) et de l'activité des com- 
munautés de sens qui en dépendent (c'est-à-dire le mode d'interaction des sujetsacteurs qui y sont impliqués, ainsi que les relations que ceux-ci entretiennent avec d'autres institutions) »

Ainsi, les communautés de sens entretiennent avec les institutions de sens peu ou prou les mêmes relations que les prédiscours vont entretenir avec les espaces interdiscursifs ; les premiers évoluent directement dans les seconds, mais de manière mutuellement non exclusive. Par ailleurs, la définition d'institution du sens telle que proposée par Sarfati fait directement écho à la théorie de l'institution soutenue par Luc Boltanski (2009 : 117/118) :

«Une institution est un être sans corps à qui est déléguée la tâche de dire ce qu'il en est de ce qui est. C'est donc (...) dans ses fonctions sémantiques qu'il faut envisager l'institution. (...) Les fonctions sémantiques des institutions dépassent largement les formes proprement linguistiques, puisqu'elles prennent en charge l'encadrement de cette gamme très large d'expressions symboliques qu'étudie la sémiotique (allant de la gestuelle à l'icône ou à la musique), comme on le voit bien dans le cas des rituels où ces différents mediums sont plus ou moins coordonnés et, dans certains cas, étroitement définis par des règles explicites »

Alors que Boltanski engage l'activité sémantique de manière large, une telle définition nous permet de repréciser, dans la lignée de Fairclough et de Paveau, que le discours reste une activité qui n'est pas exclusivement linguistique ; à ce titre, nous estimons que la théorie du sens commun développée par Sarfati permet justement d'étendre une analyse critique du discours à toutes les activités pragmatiques de la vie sociale, dans la mesure où celles-ci obéissent également à l'approche triadique représentée par le canon, la vulgate et la doxa. Ici, plus particulièrement, l'étude du niveau de la doxa (ou de l'idéologisation, en fonction des contextes) est particulièrement importante dans l'hypothèse d'une analyse critique de discours, puisque ces éléments réduits à une essence sémantique essentiellement logistique (facile à transmettre, aisément opérable pour atteindre certains buts) peuvent dissimuler en eux-mêmes les flux de simplification sémantique qui ont pour origine un canon précis. Il s'agit alors d'isoler les doxèmes (ou unités minimales de la doxa), selon Sarfati, et de porter attention à leurs mécanismes (2011: 153) :

«Une doxa s'offre toujours à la réception en situation de résultante : résultante d'un procès de diffusion sémantique d'abord marqué par l'instanciation constituante (canon), puis la transmission (vulgate). (...) L'analyse d'une doxa fait partie intégrante de la description d'une institution (ou d'une communauté) de sens, puisqu'elle suppose pour être comprise d'être située par rapport à la dynamique de sens propre à la topique dont elle constitue l'expression la moins ordonnée »

Cependant, la circulation et l'évolution de la doxa ne peuvent être analysée sans tenir compte des évolutions des institutions et des sujets eux-mêmes qui, pris dans des espaces interdiscursifs, et cognitivement enclins à mobiliser des 
prédiscours afin de pouvoir opérer de façon efficace et économique dans les situations auxquelles ils doivent faire face, sélectionnent en fonction de leurs émotions et de leur mémoire (Paveau, 2006 : 92) les atomes discursifs qui leur semblent le plus adaptés à la fois aux intérêts poursuivis, mais également au contexte en cours. Selon nous, il manque alors un élément-clé qui permettrait de comprendre la façon dont les individus (ou sujets-acteurs) peuvent laisser émerger, construire, combiner, structurer, produire et recevoir du sens. La question est donc bien la suivante : quel rôle jouent les individus lorsque les discours transitent du canon à la doxa, en passant par la vulgate, en demeurant des prédiscours mobilisables, dans un univers interdiscursifs résolument dynamique ? Et comment font les individus pour jouer ce rôle? Nous affirmons que l'inconnue qui peut permettre de résoudre cette équation des passages de sens peut être représentée par la notion de nœud sémantique (Wagener, 2015 : 189) :

« Nous postulons l'existence de nœuds sémantiques, qui permettent aux individus, de manière singulière ou collective, de s'ancrer dans le réel afin de créer des autoroutes de sens, à la manière de la métaphore connexioniste qui s'inspire des neurones et de leurs axiomes : nous avons tous plus ou moins des éléments symboliquement forts qui nous relient directement au réel et à notre façon d'y apporter du sens. Certains de ces nœuds sémantiques nous enracinent dans le réel et y apportent une valence plus 'lourde', alors que d'autres, plus proches de ballons d'air que d'ancres, nous permettent de prendre un peu de légèreté par rapport aux pressions pragmatiques de la vie quotidienne. Cette différence entre ancres et ballons permet de placer l'hypothèse des nœuds sémantiques dans un rapport non linéaire au réel, dans la mesure où il serait représenté comme une toile, à laquelle nous sommes tous reliés de façon singulière, en fonction de nos trajectoires biographiques, mais également en fonction des prédiscours collectifs et individuels disponibles et mobilisables dans notre environnement immédiat »

En ajoutant que cette toile représente l'espace interdiscursif dans lequel nous nous mouvons, conformément à la théorie des toiles de récits de Seyla Benhabib (2002 : 6/7), les nœuds sémantiques deviennent alors les points de jonction qui représentent la manière dont nous sommes reliés aux différents espaces interdiscursifs que nous investissons, ainsi qu'aux cadres prédiscursifs que nous mobilisons plus volontiers que d'autres (Wagener, 2012 : 94) :

« Ces nœuds de connexion fonctionnent comme autant de points de rencontre à partir desquels le sens se forme pour les interactants, afin que ceux-ci puissent interpréter et donner forme aux éléments à l'intérieur et à l'extérieur du système »

À partir de ces différents éléments, nous postulons le schéma suivant, qui se présente comme une extension de notre première mise en image : 


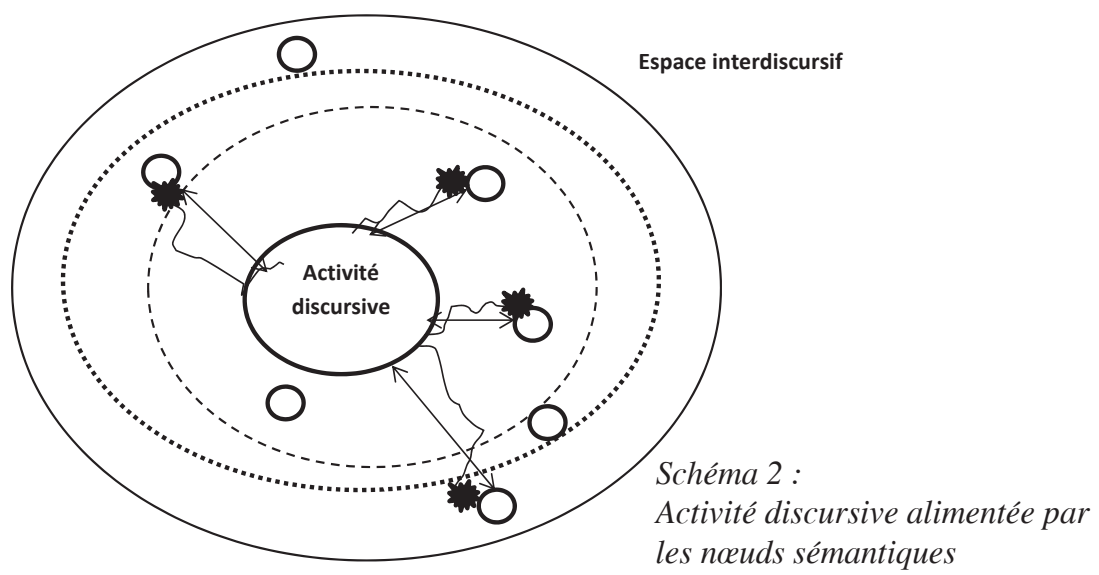

Non l'espace interdiscursif n'est pas une matérialisation mais un réseau de relations entre discours

Au sein de ce schéma, dont la représentation graphique est malheureusement limitée et nécessite d'autres approfondissements, l'activité discursive est représentée comme une émergence dynamique, reliée de façon systémique aux différents niveaux de l'espace interdiscursif, envisagé nécessairement comme un réseau de relations entre discours : le plus éloigné est celui du canon (qui nécessite un effort cognitif, éducatif et intellectuel pour pouvoir y accéder), le niveau intermédiaire représente la vulgate, et le niveau le plus proche de l'activité discursive est celui de la doxa. Bien évidemment, en fonction de l'activité discursive, nous pourrons avoir des éléments prédiscursifs mobilisés de façon complexe et interdynamique ; par exemple, il est tout à fait possible lors d'une conversation de pouvoir mobiliser à la fois un élément prédiscursif de l'ordre du canon, et d'autres de l'ordre de la doxa. C'est pour cela que nous avons souhaité représenter l'activité discursive comme reliée de manière claire à des prédiscours (représentés ici par les petits cercles vides) qui peuvent se situer sur différentes orbites de l'espace interdiscursif accessible et mobilisable pour le locuteur. D'autre part, les liens entre l'activité discursive et les prédiscours ne sont pas simplement sémantiques et praxéologiques, au sens où ils s'alimentent mutuellement, mais doivent pouvoir faire sens pour le locuteur ; c'est pour cela que chaque cadre prédiscursif est également relié à l'activité discursive par un nœud sémantique (les petites formes noires), dont le lien à l'activité est par nature irrégulier, non linéaire et peut-être même intermittent. 


\section{PERSPECTIVES : VERS UNE ANALYSE CRITIQUE PLURIDISCURSIVE.}

Notre propos a pour objectif d'embrasser la complexité du phénomène discursif, inscrit dans des expressions plurisémiotiques multiples, qui correspondent de facto à une acception large du discours, telle que nous l'avons précisée plus avant. Mais comment envisager une application méthodologique structurée d'un modèle qui cherche avant tout à rendre compte d'un phénomène complexe, dans tout ce qu'il peut avoir de désorganisé et de difficilement préhensible ? L'analyse critique pluridiscursive, c'est-à-dire capable de rassembler un corpus de discours variés et interreliés (non seulement à travers leurs interdiscours, mais également à travers leurs thématiques et leurs styles), a pour vocation de pouvoir précisément isoler les éléments discursifs selon deux axes :

- Le premier axe doit précisément préciser les éléments discursifs qui peuvent relever soit de la doxa, soit de la vulgate, soit du canon

- Le second axe doit permettre de décrire les liens entre les différents cadres prédiscursifs au sein de l'espace discursif

Ces deux dimensions sont essentielles, si l'on veut pouvoir ensuite croiser les résultats de cette double étude pour arriver à une troisième dimension de cette analyse critique, à savoir l'étude des nœuds sémantiques fondamentaux que l'on peut spécifier, au sein d'un discours construit, comme communs aux deux axes et aux différentes zones isolées entre les axes. Les aires discursives ainsi délimitées, ainsi que les récurrences discursives et leur rythme au sein d'un discours, doivent alors précisément servir à dégager les différentes topiques discursives à l'œuvre, en nous inspirant notamment des travaux d'Anscombre (1995: 191) :

« [Les topoï] sont des principes généraux qui servent d'appui au raisonnement, mais ne sont pas ce raisonnement. Ils ne sont pas assertés par leur locuteur (il prétend justement ne pas en être l'auteur), mais simplement utilisés. Ils sont par ailleurs présentés comme allant de soi au sein d'une communauté plus ou moins vaste (y compris réduite à un seul individu). C'est ce qui leur permet d'être créés de toutes pièces sans perdre un pouce d'efficacité »

En reprenant ainsi la théorie des topoï telle que décrite par Anscombre, nous revenons d'une certaine façon à l'origine des prédiscours (nous retrouvons ici l'utilisation non assertée de principes généraux) et de la doxa (la communauté utilisant des topoï allant de soi fait directement écho aux communautés de sens de Sarfati). Selon nous, un tel retour aux sources théoriques parait essentiel, dans la mesure où les discours que l' analyse critique pluridiscursive permet d'analyser ne sont pas limités aux seuls faits linguistiques, mais bien à un environnement plurisémiotique. Ceci est d'autant plus important que nous estimons ici qu'une telle analyse critique pluridiscursive devra prendre en considération une dimension importante de l'acte langagier (au sens large, donc sémiotique), 
à savoir l'argumentation - d'autant plus que notre modèle propose justement d'analyser les liens sémantiques entre les différentes aires discursives, et donc in fine la structure de ces liens. Nous souhaitons ici mobiliser les travaux de Ruth Amossy, pour qui l'argumentation est précisément au cœur du fonctionnement discursif, comme révélateur pertinent des représentations que le locuteur mobilise (en étant à la fois acteur et objet) et met en rythme afin de pouvoir donner du relief et du sens à son discours (Amossy, 2011:16) :

«L'argumentation (1) est définie comme un acte de communication où l'auditoire est primordial, et doit toujours être pris en compte ; (...) (2) se fait à travers la langue naturelle, dont elle mobilise toutes les ressources ; (3) se manifeste aussi bien sous forme de discours à visée de persuasion, que dans la dimension argumentative d'un discours qui n'entreprend pas expressément de persuader ; (...) (4) comporte un dosage variable d'appel à la raison et de recours au sentiment »

Si nous estimons toutefois que la dimension de la langue naturelle n'est pas suffisante, et qu'il faut prendre en considération toutes les variables d'expression sémiotique, nous nous devons de souligner ceci : l'analyse critique pluridiscursive doit précisément permettre de comprendre les différentes zones sémantiques que l'argumentation met en lien afin que les discours concernés puissent atteindre un certain nombre d'objectifs, de façon conscientisée ou non dans le texte (Lähdesmäki et Wagener, 2015). Ainsi, il deviendrait alors possible de ne plus simplement subir les 10\% émergés de l'iceberg discursif, mais de pouvoir explorer les $90 \%$ immergés afin de déceler les mécanismes éventuels de persuasion ou de poursuite d'intérêts pragmatiques. Pour ce faire, une analyse critique pluridiscursive se doit de rassembler un maximum d'informations sur les contextes d'énonciation des discours, pour paraphraser Cislaru et Sitri (2012 : 63), sous peine de passer complètement à côté de l'objet à analyser, ou de passer sous silence des pans entiers de sens qui demeurent inaccessibles à l'analyste qui n'aurait qu'une lecture partielle (ou pire, partiale) des discours à étudier. Cette exigence est également mise en relief par Nathalie Garric (2012 : 90/91) :

«La variation des données et des corpus constitue autant de points de vue d'interprétation des textes, elle est une forme d'insertion des textes dans l'interdiscours. Le corpus donne corps au registre, mais plus encore sa pluralisation, par les divers réseaux qu'elle engendre, permet d'établir des dynamiques dialogiques nécessaires à la discursivisation textuelle. (...) La variation, en tant que principe méthodologique, est une instrumentation de maîtrise des données hétérogènes et de manipulation de ces données pour les reconstruire en discours, eux-mêmes par définition hétérogènes. Ainsi structurées en corpus établissant des réseaux intertextuels, les données permettent de reconfigurer l'hétérogénéité en indices énonciatifs des enjeux sociaux de l'apprentissage » 
Dès lors, une analyse critique pluridiscursive se fixe plusieurs objectifs à la fois nets, complexes et résolument interconnectés :

- Délimiter la manière dont se situe une activité discursive (aux niveaux des individus, des communautés de sens et des institutions de sens)

- Comprendre la façon dont les items discursifs sont argumentés et construits entre eux, en fonction des aires sémantiques dont ils relèvent (canon, vulgate ou doxa)

- Entreprendre de dégager la rythmique inter-sémantique qui permet à des prédiscours divers et des espaces interdiscursifs plus ou moins vastes d'être mobilisés afin de faire émerger, circuler et interpréter le sens

Avant tout, la théorie de l'analyse critique pluridiscursive se veut proche d'une théorie du sens en argumentation et en expression plurisémiotique, dans la mesure où c'est pour et par le sens que les discours circulent, s'entrechoquent et s'échangent en toute intersubjectivité socialement située. En mettant l'accent sur les nœuds sémantiques, et donc sur la manière dont des éléments de sens précis nourrissent le discours et nous relient au réel, le programme de l'analyse critique pluridiscursive est de pouvoir mesurer les impacts des discours, leurs rétroactions et leurs influences mutuelles, afin de pouvoir permettre une approche didactique, nécessairement politique (au sens large du terme et non au sens militant) et volontairement ambitieuse dans une recherche de connaissance maximisée du fonctionnement du sens en discours, ainsi que du discours en sens. 


\section{BIBLIOGRAPHIE.}

Amossy, R. (2011), « Des sciences du langage aux sciences sociales : l'argumentation dans le discours », A contrario, 16 (2) : 10-25.

Anscombre, J.-C. (1995), « La théorie des topoï : sémantique ou rhétorique ? », Hermès, 15 (1) : 185-198.

Benhabib, S. (2002), The Claims of Culture. Equality and Diversity in the Global Era, Princeton : Princeton University Press.

Boltanski, L. (2009), De la critique, Paris : Gallimard.

Charaudeau, P. (1995), « Une analyse sémiolinguistique du discours », Langages, 29 (117) : 96-111.

Cislaru, G. \& Sitri, F. (2012), « De l'émergence à l'impact social des discours : hétérogénéités d'un corpus », Langages, 187 (3) : 59-72.

Fairclough, N. \& Wodak, R. (1997), "Critical Discourse Analysis”, in Van Dijk, T. A. (ed) (1997), Discourse as Social Interaction, 258-284, London: Sage.

Fairclough, N. (1999), "Global capitalism and critical awareness of language", Language Awareness, 8 (2) : 71-83.

Fairclough, N. (2005), "Discourse analysis in organizational studies: the case for critical realism”, Organization Studies, 26 (6) : 915-939.

Galatanu, O. (2009), «L'analyse du discours dans la perspective de la sémantique des possibles argumentatifs : les mécanismes sémantico-discursifs de construction du sens et de reconstruction de la signification lexicale », in Garric, N. \& Longhi, J. (ed) (2009), L'analyse linguistique des corpus discursifs. Des théories aux pratiques, des pratiques aux théories, 49-68, Clermont-Ferrand : Presses Universitaires Blaise-Pascal.

Garric, B. \& Capdevielle-Mougnibas, V. (2009), « La variation comme principe d'exploration de corpus : intérêts et limites de l'analyse lexicométrique interdiciplinaire pour l'étude de discours », Corpus, 8 : 105-128.

Garric, N. (2012), « Construire et maîtriser l'hétérogénéité par la variation des données, des corpus et des méthodes », Langages, 187 (3) : 73-92.

Garric, N. \& Longhi, J. (2013), « Atteindre l'Interdiscours par la Circulation des Discours et du Sens », Langage et Société, 144 (2) : 65-83.

Lähdesmäki, T. \& Wagener, A. (2015), "Discourses on governing diversity in Europe : critical analysis of the White Paper on Intercultural Dialogue", International Journal of Intercultural Relations, 44 : 13-28.

Longhi, J. (2009), « Les objets discursifs et le phénomène d'anticipation lexicale du discours : processus de référenciation et argumentativité dans l'activité discursive », in Garric, N. \& Longhi, J. (eds), L'analyse linguistique des corpus discursifs. Des 
théories aux pratiques, des pratiques aux théories, 69-92, Clermont-Ferrand : Presses Universitaires Blaise-Pascal.

Pêcheux, M. (1975), Les Vérités de La Palice, Paris : Maspero.

Paveau, M.-A. (2006), Les prédiscours: sens, mémoire, cognition, Paris : Presses Sorbonne Nouvelle.

Sarfati, G.-E. (2007), « Note sur 'sens commun' : essai de caractérisation linguistique et sociodiscursive », Langage et société, 119 (1) : 63-80.

Sarfati, G.-E. (2008), « Pragmatique linguistique et normativité : remarques sur les modalités discursives du sens commun », Langages, 170 (2) : 92-108.

Sarfati, G.-E. (2011), « Analyse du discours et sens commun : institutions de sens, communautés de sens, doxa, idéologie », in Guilhaumou, J. \& Schepens, P. (eds.). Matériaux philosophiques pour l'analyse du discours, 139-174, Besançon : Presses Universitaires de Franche-Comté.

Van Dijk, T. (1993), "Principles of critical discourse analysis", Discourse and society, 4 (2) : 249-283.

Wagener, A. (2012), «Connexions sémantiques et contextique relationnelle : pour une modélisation complexe des interactions humaines », Nouvelles Perspectives en Sciences Sociales, 7 (2) : 67-104.

Wagener, A. (2015), L'échec culturel, Bruxelles : Peter Lang. 